\title{
Respostas Fisiológicas e Perceptuais \\ Obtidas Durante a Caminhada em Ritmo \\ Autosselecionado por Mulheres com \\ Diferentes Î́ndices de Massa Corporal
}

\section{Physiological and Perceived Responses Obtained during Gait in Self-Selected Rhythm by Women with Different Body Mass Indices}

\author{
Hassan M. Elsangedy \\ Kleverton Krinski \\ Cosme F. Buzzachera ${ }^{2}$ \\ Renan F. H. Nunes ${ }^{1}$ \\ Flavia A. M. Almeida' \\ Carlo Baldari² \\ Laura Guidetti² \\ Wagner de Campos ${ }^{1}$ \\ Sergio G. da Silva \\ 1. Centro de Pesquisa em Exercício e \\ Esporte - CEPEE. \\ Universidade Federal do Paraná, \\ Curitiba,PR, Brasil. \\ 2. Universidade de Estudos de Roma \\ Foro Italico, Roma, Itália.

\section{Endereço para correspondência: \\ Sergio Gregorio da Silva \\ Rua Coração de Maria, 92 - \\ Jd. Botânico \\ 80215-370 - Curitiba, PR - Brasil \\ Tel.: (41) 3360-4331 \\ Fax: (41) 3360-4336 \\ E-mail: sergiogregorio@ufpr.br}

Submetido em 03/07/2008

Versão final recebida em 29/01/2009

Aceito em 30/01/2009

\begin{abstract}
RESUMO
As respostas fisiológicas e perceptuais verificadas durante a caminhada vêm sendo amplamente pesquisadas; contudo, poucas investigações buscaram comparar essas respostas em grupos de diferentes índices de massa corporal durante caminhada em ritmo autosselecionado. O objetivo do presente estudo foi comparar as respostas fisiológicas e perceptuais entre mulheres com peso normal (PN), sobrepeso (SP) e obesas (OB) durante a caminhada em ritmo autosselecionado. Participaram 66 mulheres sedentárias, com idade entre 20 e 45 anos, designadas em três grupos de acordo com o seu índice de massa corporal: (i) PN $\left(18,5-24,9 \mathrm{~kg} \cdot \mathrm{m}^{-2}, \mathrm{n}=22\right)$; (ii) SP $\left(25,0-29,9 \mathrm{~kg} \cdot \mathrm{m}^{-2}, \mathrm{n}=22\right)$; e (iii) $\mathrm{OB}\left(\geq 30,0 \mathrm{~kg} \cdot \mathrm{m}^{-2}, \mathrm{n}=22\right)$. Cada participante realizou uma sessão de familiarização e duas sessões experimentais (teste incremental máximo e teste de 20 minutos de caminhada em ritmo autosselecionado), a fim de determinar as respostas fisiológicas (frequência cardíaca, FC; e consumo de oxigênio, VO 2 ) e perceptuais (percepção subjetiva de esforço, PSE) de cada sessão. A ANOVA one-way foi empregada para comparar as respostas fisiológicas e perceptuais obtidas durante a caminhada em ritmo autosselecionado entre os três protocolos experimentais, empregando-se o teste post hoc de Tukey com $p<0,05$. Embora os sujeitos com obesidade tenham registrado menor velocidade de caminhada $\left(1,30 \pm 0,13 \mathrm{~m} \cdot \mathrm{s}^{-1}\right)$ comparativamente aos com peso normal e sobrepeso $(1,69 \pm 0,11$ e 1,58 \pm $0,22 \mathrm{~m} \cdot \mathrm{s}^{-1}$, respectivamente) $(p<0,05)$, eles apresentaram similar resposta fisiológica $(58,7 \pm 8,6 ; 63,1 \pm 12,6$; $64,8 \pm 11,1 \% \mathrm{VO}_{2}$ e $75,1 \pm 7,8 ; 78,8 \pm 8,5 ; 78,7 \pm 7,2 \% \mathrm{FC}_{\text {max }}$ para os grupos normal, sobrepeso e obeso, respectivamente). Além disso, as respostas perceptuais também não diferiram entre os grupos experimentais $(11,6 \pm 1,6 ; 11,7 \pm 1,6 ; 12,2 \pm 1,8$, respectivamente). Desse modo, pode-se concluir que, independente do IMC, mulheres adultas apresentam respostas fisiológicas e perceptuais similares durante caminhada em ritmo autosselecionado, mesmo caminhando em velocidades diferentes.
\end{abstract}

Palavras-chave: obesidade, atividade física, esforço.

\section{ABSTRACT}

Physiological and perceived responses verified during gait have been widely studied; however, few investigations have tried to compare these responses in groups of different body mass indices during gait in self-selected rhythm. The aim of the present study was to compare the physiological and perceived responses among normative $(\mathrm{N})$, overweighed $(\mathrm{OW})$ and obese $(\mathrm{OB})$ women during gait in self-selected rhythm. Sixty-six sedentary women, aged between 20 and 45 years and divided in three groups according to their body mass indices participated in the study: (i) $\mathrm{N}\left(18.5-24.9 \mathrm{~kg} \cdot \mathrm{m}^{-2}, \mathrm{n}=22\right)$; (ii) OW (25.0 - $\left.29.9 \mathrm{~kg} \cdot \mathrm{m}^{-2}, \mathrm{n}=22\right)$ and (iii) OB $\left(\geq 30.0 \mathrm{~kg} \cdot \mathrm{m}^{-2}, \mathrm{n}=22\right.$ ). Each participant performed a familiarization session and two experimental sessions (maximal incremental test and 20m-walking test in self-selected rhythm) to determine the physiological (heart rate - HR and oxygen consumption - $\mathrm{O}_{2}$ ) and perceived responses (perceived exertion - PE) of each session. One-way ANOVA was applied to compare the physiological and perceived responses obtained during gait in self-selected rhythm among the three experimental protocols, applying the Tukey's post hoc test with $p<0.05$. Although the obese subjects have registered lower walking velocity $\left(1.30 \pm 0.13 \mathrm{~m} \cdot \mathrm{s}^{-1}\right)$ compared with the normative and overweighed ones $\left(1.69 \pm 0.11\right.$ and $1.58 \pm 0.22 \mathrm{~m} \cdot \mathrm{s}^{-1}$, respectively) $(p<0.05)$, they presented similar physiological response $\left(\left(58.7 \pm 8.6 ; 63.1 \pm 12.6 ; 64.8 \pm 11.1 \% \mathrm{O}_{2}\right.\right.$ and $75.1 \pm 7.8 ; 78.8 \pm 8.5$; $78.7 \pm 7.2 \% \mathrm{HR}_{\max }$ for the normative, overweighed and obese groups, respectively). Moreover, the perceived responses did not differ between experimental groups (11.6 $\pm 1.6 ; 11.7 \pm 1.6 ; 12.2 \pm 1.8$, respectively). Therefore, it can be concluded that regardless of the BMI, adult women present similar physiological and perceived responses during gait in self-selected rhythm even when walking in different velocities.

Keywords: obesity, physical activity, exertion. 


\section{INTRODUÇÃO}

O índice de massa corporal (IMC) acima de $25 \mathrm{~kg} \cdot \mathrm{m}^{-2}$ têm sido associado a maior risco para o desenvolvimento de doenças cardiovasculares ${ }^{(1)}$. No Brasil, aproximadamente $40 \%$ dos indivíduos adultos apresentam sobrepeso (SP) por esse critério (2). No intuito de amenizar ou reduzir esse quadro, estratégias têm sido sugeridas, como a caminhada, devido a sua simplicidade e segurança ${ }^{(3,4)}$, propiciando um meio adequado para redução do peso corporal e melhora do condicionamento cardiorrespiratório(5). Para alcançar esses benefícios, quantidade e intensidade apropriadas de exercício físico são necessárias ${ }^{(4)}$. Diretrizes para prática de exercícios físicos recomendam para a melhoria da aptidão cardiorrespiratória 60-90 minutos de exercício, com intensidade entre 50 e 85\% do consumo máximo de oxigênio $\left(\mathrm{VO}_{2 \text { máx }}\right), 55-90 \%$ da frequência cardíaca máxima e percepção subjetiva do esforço (PSE) entre 12 e 16, na maioria dos dias da semana(4,6).

Apesar da importância de um adequado estímulo fisiológico, Dishman et al. ${ }^{(3)}$ demonstraram tendência de os participantes de atividades físicas programadas buscarem exercitar-se em intensidade autosselecionada em detrimento de uma intensidade prescrita. Os estudos que buscaram investigar a efetividade da caminhada em intensidade de autosselecionada, em sua maioria, utilizaram mulheres sedentárias com peso normal $(\mathrm{PN})^{(3,7)}$. Os poucos estudos que avaliaram a influência do peso corporal sobre a intensidade de caminhada compararam apenas sujeitos com PN e SP(8,9), negligenciando a obesidade. Em estudo singular, Hills et al. ${ }^{(10)}$ compararam sujeitos PN e obesos (OB); contudo, mensuraram apenas a resposta da FC para determinar a intensidade do exercício.

Em relação ao controle da intensidade do exercício selecionado, Johnson e Phipps ${ }^{(11)}$ relataram que a maioria dos sujeitos utiliza a percepção do esforço, em virtude de esse método ser acessível e apresentar ampla aplicabilidade, dispensando equipamentos específicos e/ou extensiva experiência dos sujeitos, permitindo quantificar os sinais oriundos dos sistemas cardiorrespiratório e neuromuscular durante o exercício ${ }^{(12,13)}$.

A PSE tem sido utilizada para mensurar a percepção do esforço em diversas populações $(3,4,7,8,8,9,10,12,13)$; contudo, poucas investigações buscaram comparar essas respostas em grupos de diferentes massas corporais em ritmo autosselecionado $(3,4,7,8,9)$. Os estudos realizados por Ekkekakis e Lind ${ }^{(8)}$ e Pintar et al.(9), ao comparar as respostas perceptuais em indivíduos com PN e SP, observaram respostas similares entre os grupos durante a caminhada em ritmo autosselecionado. Contudo, ambos os estudos não avaliaram indivíduos OB que, segundo Mattsson et al.(14), exibem menor tolerância à intensidade do exercício com tendência a reportar maior PSE em virtude das mudanças estruturais e funcionais causadas pelo excesso de peso corporal. Controversamente, Hills et al. ${ }^{(10)}$ reportaram não haver diferenças para PSE entre $\mathrm{OB}$ e PN durante o exercício em ritmo autosselecionado.

Considerando as explanações acima, fica evidente a escassez de informações e os resultados conflitantes existentes sobre as respostas fisiológicas e perceptuais durante a caminhada em ritmo autosselecionado entre sujeitos com diferentes índices de massa corporal. Até o presente momento, nenhum estudo buscou comparar em conjunto as respostas fisiológicas e perceptuais de sujeitos PN, SP e OB durante a caminhada em ritmo autosselecionado. Dessa maneira, o objetivo do presente estudo foi comparar as respostas fisiológicas e perceptuais entre mulheres com peso normal, sobrepeso e obesas durante a caminhada em ritmo autosselecionado.

\section{MÉTODOS}

\section{Sujeitos}

Participaram do presente estudo 66 mulheres sedentárias, com idade de 20 a 45 anos, as quais foram posteriormente alocadas para um dos três grupos experimentais, de acordo com o seu IMC: (a) peso normal (PN, 18,5-24,9kg $\left.\cdot \mathrm{m}^{-2}, n=22\right)$, (b) sobrepeso (SP, 25,0-29,9kg. $\mathrm{m}^{-2}$, $n=22)$, e (c) obeso $\left(\mathrm{OB},>30,0 \mathrm{~kg} \cdot \mathrm{m}^{-2}, n=22\right)$, conforme os pontos de corte estabelecido pela Organização Mundial de Saúde ${ }^{(15)}$. O tamanho da amostra foi determinado utilizando nível de significância de 0,05, poder estatístico de 0,70 e magnitude de efeito de 0,35, indicando 22 sujeitos para cada grupo experimental. Para participar do presente estudo, os sujeitos deveriam ser não fumantes, sem problemas clínicos, neuromotores ou cognitivos que contraindicassem a prática de exercício físico, ou fizessem uso de medicação que influenciasse as funções metabólicas ou cognitivas, verificadas em avaliação médica. As características físicas e antropométricas dos sujeitos são apresentadas nas tabelas 1 e 2 . Durante a avaliação inicial, cada participante leu e assinou o termo de consentimento após as explicações do objetivo, procedimentos experimentais, possíveis riscos e benefícios inerentes à pesquisa, conforme as diretrizes propostas na Resolução 196/96 do Conselho Nacional de Saúde sobre pesquisas envolvendo seres humanos. O presente estudo foi aprovado pelo Comitê de Ética em Pesquisa do Setor de Ciências Biológicas da Universidade Federal do Paraná (Protocolo n 530.067.08.05).

\section{Variáveis antropométricas}

As variáveis antropométricas, massa corporal (MC, em kg; balança marca Toledo, modelo 2096, São Paulo, Brasil), estatura (EST, em cm; estadiômetro marca Sanny, modelo Standard, São Bernardo do Campo, Brasil), foram obtidas e, posteriormente, o IMC $\left(\mathrm{kg} \cdot \mathrm{m}^{-2}\right)$ foi calculado pela razão entre MC e a EST ao quadrado. A densidade corporal foi mensurada através do método de espessura de dobras cutâneas (bicipital, tricipital, subescapular e suprailíaca), de acordo com a equação proposta por Durnin e Womersley ${ }^{(16)}$. Posteriormente, o percentual de gordura corporal (\%gord) foi obtido mediante utilização da equação de Siri ${ }^{(17)}$. Buscando evitar variações interavaliadores, todas as medidas foram obtidas por um único avaliador previamente treinado.

\section{Sessão de familiarização}

Os sujeitos foram familiarizados com a utilização da esteira e aparatos do analisador de gases, recebendo instruções padronizadas sobre o uso da escala de PSE(18). Subsequentemente, foram esclarecidos os procedimentos para ajuste da velocidade através de sensores acoplados a esteira, que foi testado pelos sujeitos durante a execução de 10 minutos de caminhada em ritmo autosselecionado, iniciando a uma velocidade de 1,11 m:s $\mathrm{s}^{-1}$ sem inclinação. O ritmo autosselecionada da caminhada foi definido como a velocidade em que o sujeito considerava confortável para uma estipulada duração da atividade ${ }^{(9)}$.

\section{Teste incremental em esteira}

Após um intervalo de 48 horas em relação à sessão de familiarização, todos os participantes realizaram aquecimento em esteira (marca Reebok Fitness, modelo X-fit 7, Londres, Reino Unido) de cinco minutos a uma velocidade padrão de $1,11 \mathrm{~m} \cdot \mathrm{s}^{-1}$ sem inclinação. Posteriormente, um teste de esteira incremental foi conduzido mediante utilização de protocolo estabelecido por Lind et al. ${ }^{(7)}$, iniciando com velocidade de $1,11 \mathrm{~m} \cdot \mathrm{s}^{-1} \mathrm{sem}$ inclinação por dois minutos, aumentando $0,18 \mathrm{~m} \cdot \mathrm{s}^{-1} \mathrm{a}$ cada minuto até a exaustão.

Durante essa sessão, a mensuração do consumo de oxigênio $\left(\mathrm{VO}_{2}\right)$, produção de dióxido de carbono $\left(\mathrm{VCO}_{2}\right)$ e ventilação pulmonar (VE) foram obtidos através de um sistema de espirometria computadorizado de circuito aberto (True Max 2400, Parvo Medics", Salt Lake City, UT, EUA), que foi calibrado antes de cada teste usando o ar ambiente e um gás com concentrações conhecidas de $\mathrm{O}_{2}$ e $\mathrm{CO}_{2}$, enquanto a turbina bidirecional foi calibrada usando uma seringa de 3-L (marca Hans Rudolph ${ }^{\oplus}$, modelo 5530, Kansas City, Missouri, EUA). Para que o valor de $\mathrm{VO}_{2 \text { máx }}$ fosse aceito, os participantes deveriam alcançar um dos seguintes critérios: (a) platô do $\mathrm{VO}_{2}$, indicado por diferença $<150 \mathrm{~mL} \cdot \mathrm{min}^{-1}$; (b) razão de troca respiratória $\geq 1,10$; e (c) e frequência cardíaca dentro de $\pm 10 \mathrm{bpm}$ da $F C_{\text {máx }}$ predita pela idade para cada sujeito.

A FC foi obtida a cada cinco segundos utilizando um cardiofrequêncimetro (Polar Electro ${ }^{\oplus}$, Oy, Finlândia). A FC máx foi determinada como a maior FC média (intervalo de um minuto) verificada no último estágio completo do teste incremental. 
Teste de 20 minutos de caminhada em ritmo autosselecionado em esteira

Anteriormente à realização do teste, todas as instruções referentes à autosseleção da velocidade e utilização da escala de PSE foram repassadas. Inicialmente, foi conduzido um aquecimento de cinco minutos em velocidade de $1,11 \mathrm{~m} \cdot \mathrm{s}^{-1} \mathrm{sem}$ inclinação. Na sequência, os sujeitos foram instruídos a ajustar um ritmo autosselecionado, conforme os procedimentos propostos por Pintar et al. ${ }^{(9)}$, mediante a utilização de sensores de controle de velocidade acoplados à esteira. $O$ teste iniciou-se à velocidade de $1,11 \mathrm{~m} \cdot \mathrm{s}^{-1} \mathrm{sem}$ inclinação, sendo permitido aos sujeitos o ajuste da velocidade de maneira livre, a fim de que buscassem um ritmo considerado confortável para um período de 20 minutos de exercício. Os ajustes foram realizados durante o primeiro minuto de caminhada e, posteriormente, somente nos minutos 00:05, 00:10 e 00:15. O marcador de velocidade foi ocultado para o avaliado, conforme proposto por Pintar et al. ${ }^{(9)}$. As variáveis fisiológicas e perceptuais foram determinadas utilizando os mesmos instrumentos e procedimentos do teste incremental em esteira; contudo, foram considerados somente os valores dos minutos 00:05, 00:10, 00:15 e 00:20(7); as respostas fisiológicas foram reduzidas para médias dos últimos dois minutos em intervalos de 15 segundos dos respectivos estágios ${ }^{(9}$; em seguida, foram calculadas médias gerais utilizando esses valores para representar os 20 minutos de caminhada em cada grupo para posterior análise estatística.

\section{Percepção subjetiva do esforço}

A percepção subjetiva de esforço (PSE) foi mensurada através da Escala de Esforço Percebido (6-20)(12); a definição, as instruções sobre o uso da escala e procedimentos de ancoragem foram explanados imediatamente antes dos testes, seguindo os procedimentos propostos por Robertson et al. ${ }^{(18)}$. A ancoragem da baixa (7 - menor intensidade de exercício) e alta (19 - maior intensidade de exercício) percepção de esforço foram determinadas no teste incremental máximo. A escala foi administrada durante os últimos 15 segundos de cada estágio de um minuto no teste incremental máximo e no teste de caminhada em esteira de 20 minutos, considerando para análise o valor obtido nos minutos 00:05, 00:10, 00:15 e 00:20.

\section{Procedimentos estatísticos}

Os dados descritivos são apresentados como média (M) e desvio padrão (DP). A ANOVA one-way (IMC) foi utilizada para comparar as variáveis antropométricas e as respostas fisiológicas e perceptuais dos grupos experimentais (PN, SP, OB) obtidas no teste incremental máximo e teste de caminhada de 20 minutos. O teste post hoc de Tukey foi empregado para localizar as diferenças entre os grupos. Todas as análises foram realizadas mediante a utilização do SPSS for Windows, versão 13.0, considerado nível de significância de $p<0,05$.

\section{RESULTADOS}

A ANOVA one-way revelou diferenças entre os grupos PN, SP e OB para massa corporal $\left(F_{2,63}=79,835, p<0,001\right)$, IMC $\left(F_{2,63}=129,156, p<\right.$ $0,001)$, massa livre de gordura $\left(F_{2,63}=53,044, p<0,001\right)$ e \%gord $\left(F_{2,63}\right.$ $=20,897, p<0,001)$. Contudo, não foram verificadas diferenças significantes para a idade e estatura $(p>0,05)$ (tabela 1).

A ANOVA one-way confirmou que no teste incremental máximo não houve diferenças nas respostas fisiológicas e perceptuais $(p>0,05)$, com exceção do $V_{2 \text { máx }}\left(F_{2,63}=499,021, p=0,001\right)$, que foi maior no grupo PN comparado com os grupos SP e OB. A velocidade máx também se demonstrou diferente entre os três grupos $\left(F_{2,63}=23,780, p=0,001\right)$, sendo menor no grupo OB comparado com os demais (tabela 2).

As respostas fisiológicas e perceptuais do teste de 20 minutos de caminhada em ritmo autosselecionado em esteira são apresentadas na tabela 3. A ANOVA one-way indicou que somente o VO $\mathrm{V}_{2}$ absoluto $\left(F_{2,63}\right.$ $=5,921, p=0,005)$ e velocidade de caminhada $\left(F_{2,63}=25,977, p=0,001\right)$ foram diferentes entre os grupos; ambas as variáveis foram menores no grupo OB. As respostas perceptuais não apresentaram diferenças entre os grupos experimentais $(p>0,05)$.
Tabela 1. Características antropométricas dos participantes.

\begin{tabular}{l|c|c|c}
\hline & $\begin{array}{c}\text { Peso normal } \\
(n=22)\end{array}$ & $\begin{array}{c}\text { Sobrepeso } \\
(n=22)\end{array}$ & $\begin{array}{c}\text { Obesas } \\
(n=22)\end{array}$ \\
\hline Idade (anos) & $30,8 \pm 9,3$ & $34,8 \pm 8,6$ & $33,5 \pm 8,5$ \\
\hline Massa corporal $(\mathrm{kg})$ & $58,5 \pm 6,8$ & $68,3 \pm 8,8^{*}$ & $89,3 \pm 8,9^{\text {\# }}$ \\
\hline Estatura $(\mathrm{cm})$ & $162,6 \pm 7,0$ & $160,2 \pm 7,4$ & $160,1 \pm 5,6$ \\
\hline IMC $\left(\mathrm{kg} \cdot \mathrm{m}^{-2}\right)$ & $22,0 \pm 1,6$ & $26,4 \pm 1,3^{*}$ & $34,9 \pm 4,1^{\text {\# }}$ \\
\hline MLG & $42,4 \pm 3,1$ & $47,4 \pm 5,5^{*}$ & $57,9 \pm 5,5^{\text {\# }}$ \\
\hline \%gord & $26,9 \pm 4,9$ & $30,8 \pm 2,7^{*}$ & $34,5 \pm 3,1^{\text {\# }}$ \\
\hline
\end{tabular}

Dados em média \pm DP. IMC: índice de massa corporal; MLG: massa livre de gordura. * Significante diferença entre os grupos com peso normal e sobrepeso $(p<0,05)$. "Significante diferença entre o grupo com peso normal e obeso $(p<0,05)$. ${ }^{\dagger}$ Significante diferença entre o grupo com sobrepeso e obeso $(p<0,05)$.

Tabela 2. Respostas fisiológicas, perceptuais e velocidade dos participantes.

\begin{tabular}{l|c|c|c}
\hline & $\begin{array}{c}\text { Peso normal } \\
(n=22)\end{array}$ & $\begin{array}{c}\text { Sobrepeso } \\
(n=22)\end{array}$ & $\begin{array}{c}\text { Obesas } \\
(n=22)\end{array}$ \\
\hline $\mathrm{VO}_{2 \text { máx }}\left(\mathrm{ml} \cdot \mathrm{kg}^{-1} \cdot \mathrm{min}^{-1}\right)$ & $34,8 \pm 7,5$ & $31,7 \pm 5,1$ & $25,5 \pm 4,1^{\#+}$ \\
\hline $\mathrm{FC}_{\text {máx }}\left(\mathrm{bp} \cdot \mathrm{min}^{-1}\right)$ & $184,6 \pm 12,0$ & $179,7 \pm 14,4$ & $180,7 \pm 9,1$ \\
\hline $\mathrm{VE}\left(\mathrm{L} \cdot \mathrm{min}^{-1}\right)$ & $57,7 \pm 10,4$ & $61,3 \pm 9,6$ & $64,1 \pm 9,7$ \\
\hline $\mathrm{RER}$ & $1,16 \pm 0,06$ & $1,13 \pm 0,07$ & $1,17 \pm 0,06$ \\
\hline PSE & $12,1 \pm 1,9$ & $12,0 \pm 1,7$ & $12,1 \pm 1,2$ \\
\hline$V_{\mathrm{LV}}(6-20)$ & $2,94 \pm 0,52$ & $2,66 \pm 0,36$ & $2,08 \pm 0,27^{\text {\# }}$
\end{tabular}

Dados em média $\pm \mathrm{DP}$. VO $\mathrm{O}_{2 \text { maxi }}$ consumo máximo de oxigênio; $F C_{\text {maxx }}$ frequência cardíaca máxima; E: ventilaçăo pulmonar; RER: coeficiente respiratório; PSE: taxa de percepçăo do esforço; LV: limiar ventilatório. "Significante diferença entre os grupos com peso normal e sobrepeso $(p<0,05)$. "Significante diferença entre o grupo com peso normal e obeso $(p<0,05){ }^{\dagger}$ Significante diferença entre o grupo com sobrepeso e obeso $(p<0,05)$.

Tabela 3. Respostas fisiológicas, perceptuais e velocidade durante caminhada em ritmo autosselecionado.

\begin{tabular}{l|c|c|c}
\hline & $\begin{array}{c}\text { Peso normal } \\
(N=22)\end{array}$ & $\begin{array}{c}\text { Sobrepeso } \\
(N=22)\end{array}$ & $\begin{array}{c}\text { Obesas } \\
(N=22)\end{array}$ \\
\hline $\mathrm{VO}_{2}\left(\mathrm{ml} \cdot \mathrm{kg}^{-1} \cdot \mathrm{min}^{-1}\right)$ & $20,0 \pm 3,49$ & $20,0 \pm 4,6$ & $16,6 \pm 2,6^{\sharp+}$ \\
\hline $\mathrm{VO}_{2 \text { máx }}$ & $58,7 \pm 8,6$ & $63,1 \pm 12,6$ & $64,8 \pm 11,1$ \\
\hline $\mathrm{FC}\left(\mathrm{bp} \cdot \mathrm{min}^{-1}\right)$ & $138,6 \pm 16,2$ & $141,1 \pm 14,9$ & $141,2 \pm 14,8$ \\
\hline$\% F C_{\text {máx }}$ & $75,1 \pm 7,8$ & $78,8 \pm 8,5$ & $78,7 \pm 7,2$ \\
\hline PSE $(6-20)$ & $11,6 \pm 1,6$ & $11,7 \pm 1,6$ & $12,2 \pm 1,8$ \\
\hline Velocidade $\left({\left.\mathrm{m} \cdot \mathrm{s}^{-1}\right)}^{\text {Del }}\right.$ & $1,69 \pm 0,11$ & $1,58 \pm 0,22$ & $1,30 \pm 0,13^{\# \dagger}$ \\
\hline
\end{tabular}

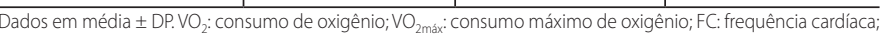

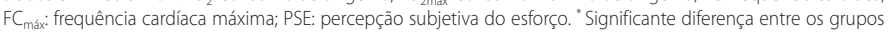
com peso normal e sobrepeso $(p<0,05)$. "Significante diferença entre o grupo com peso normal e obeso ( $p<$ $0,05)$. ${ }^{\dagger}$ Significante diferença entre o grupo com sobrepeso e obeso $(p<0,05)$.

\section{DISCUSSÃO}

O entendimento de como o IMC influência as respostas fisiológicas e perceptuais durante a caminhada em ritmo autosselecionado poderia contribuir para futuros delineamentos experimentais na área de estudo do exercício físico realizado em intensidade autosselecionada, especialmente entre indivíduos com diferentes IMC. Dessa maneira, a proposta do presente estudo foi examinar as respostas fisiológicas e perceptuais em mulheres com peso normal, sobrepeso e obesas durante caminhada em ritmo autosselecionado.

Na presente investigação, foi observado que a velocidade de caminhada em intensidade autosselecionada foi similar entre os grupos PN e SP; entretanto, ambos os grupos apresentaram maior velocidade de caminhada que o grupo OB. Similarmente, Pintar et al.(9) e Ekekkakis e Lind ${ }^{(8)}$ demonstraram não haver diferenças significantes entre os grupos PN $\left(1,47 \mathrm{~m} \cdot \mathrm{s}^{-1}\right.$ e $1,80 \mathrm{~m} \cdot \mathrm{s}^{-1}$, respectivamente) e SP $\left(1,38 \mathrm{~m} \cdot \mathrm{s}^{-1}\right.$ e $1,52 \mathrm{~m} \cdot \mathrm{s}^{-1}$, respectivamente) durante a caminhada em ritmo autosselecionado. Os achados do presente estudo para o grupo OB corroboram os encontrados por Mattsson et al. ${ }^{(14)}$, os quais reportaram que o grupo OB caminhou mais lentamente $\left(1,16 \mathrm{~m} \cdot \mathrm{s}^{-1}\right)$ comparado com o grupo PN $\left(1,30 \mathrm{~m} \cdot \mathrm{s}^{-1}\right)$. De forma similar, Hills et 
al. ${ }^{(10)}$, observaram que mulheres $O B$ adotam menor velocidade de caminhada que o grupo PN em intensidade autosselecionada. Essas evidências demonstram que sujeitos com PN e SP tendem a autosselecionar similar velocidade de caminhada; contudo, sujeitos OB buscam caminhar em velocidade inferior comparado com seus pares, possivelmente em virtude das mudanças estruturais e funcionais causadas pelo excesso de peso corpora|(14).

Contudo, apesar das diferenças na velocidade entre os grupos PN e SP com o grupo OB, as respostas fisiológicas ( $\% \mathrm{VO}_{2 \text { máx }}$ e $\left.\% \mathrm{FC}_{\text {máx }}\right)$ para os 20 minutos de caminhada foram consistentes com a transição entre intensidade de exercício "moderada" e "vigorosa", excedendo o limiar mínimo (55-65\% FC $\mathrm{C}_{\text {máx }}$ e 40-50\% $\mathrm{VO}_{2 \text { máx }}$ ) recomendado para melhora do condicionamento físico ${ }^{(4)}$. Esses resultados estão de acordo com os achados de Pintar et al. (9), que ao comparar mulheres com PN $(n=15)$ e com SP $(n=15)$, ambas com baixo condicionamento $\left(\mathrm{VO}_{2 \operatorname{máx}}\right.$ de 30,4 \pm 4,2 e 30,9 $\pm 4,9$, respectivamente), durante caminhada em ritmo autosselecionado, não verificaram diferenças para \%VO ${ }_{2 \text { máx }}(54,4 \pm 15,4$ e 51,4 $\pm 12,4$, respectivamente) e $\% F C_{\text {máx }}(67,6 \pm 11,0$ e 70,2 $\pm 11,5$, respectivamente). Entretanto, estudo realizado por Ekkekakis e Lind ${ }^{(8)}$, ao avaliar mulheres com PN $(n=9)$ e SP $(n=16)$ durante caminhada em ritmo autosselecionado, demonstrou que o grupo SP utilizou maior \% $\mathrm{VO}_{2 \text { máx }}$. Essas diferenças encontradas entre esses estudos são esperadas, vista a discrepância verificada na velocidade de caminhada.

Para o grupo $\mathrm{OB}$, as respostas fisiológicas verificadas no presente estudo estão em contraste com os encontrados por Hills et al. (10), que demonstraram maior resposta da FC (+15bpm) no grupo OB (correspondendo a $70 \% \mathrm{FC}_{\text {máx }}$ ) comparado com o PN quando instruídos a "caminhar com prazer." Na pesquisa realizada por Hills et al. ${ }^{(10)}$, embora tenham avaliado mulheres obesas, a definição de obesidade foi pautada no percentual de gordura e não IMC e foi utilizado apenas a FC para mensurar a intensidade do exercício. Além disso, uma significante diferença foi verificada para idade entre os grupos, que poderia ter influenciado a intensidade relativa do exercício ${ }^{(19)}$. De acordo com Browning et al.(20), indivíduos OB tendem buscar velocidade de caminhada preferida inferior à de indivíduos com PN e SP, no intuito de reduzir o custo energético pela distância. No presente estudo verificamos que. embora o grupo OB tenha autosselecionado uma velocidade de caminhada inferior à verificada nos outros grupos, os parâmetros fisiológicos $\left(\% \mathrm{VO}_{2 \text { máx }} \mathrm{e} \% \mathrm{FC}_{\text {máx }}\right)$ demonstram valores semelhantes aos apresentados pelo grupo PN e SP; esses achados podem ser em decorrência de ineficiência biomecânica da caminhada ${ }^{(20)}$.

No presente estudo, as respostas psicobiológicas mensuradas pela PSE foram similares entre os grupos (PSE 11). Esses resultados contrastam com os achados por Ekkekakis e Lind ${ }^{(8)}$, que observaram diferentes níveis de PSE para o grupo SP comparado com o PN. Entretanto, essas diferenças são possivelmente em consequência da intensidade do exercício, que foi maior no grupo com SP comparado com PN. Contudo, Pintar et al.(9) verificaram resultados similares aos achados do

\section{REFERÊNCIAS BIBLIOGRÁFICAS}

1. $\mathrm{NIH}$ - National Institutes of Health. Clinical guidelines on the identification, evaluation, and treatment of overweight and obesity in adults: the evidence report. Obes Res. 1998; 6 Suppl 2:51S-209S.

2. IBGE. Instituto Brasileiro de Geografia e Estatística. Pesquisa de orçamentos familiares - POF 2002 - 2003. Disponivel em: http://www.ibge.gov.br/home/ presidencia/noticias /noticia_visualiza.php?id_noticia=278. Acessado em: 17/06/2008

3. Dishman RK, Farquhar RP, Cureton KJ. Responses to preferred intensities of exertion in men differing in activity levels. Med Sci Sports Exerc. 1994; 26(6):783-90.

4. American College of Sports Medicine. ACSM's guidelines for exercise testing and prescription, seventh ed. Philadelphia: Lippincott Williams and Wilkins, 2006

5. Jakicic JM, Winters C, Lang W, Wing RR. Effects of intermittent exercise and use of home exercise equipment on adherence, weight loss, and fitness in overweight women: a randomized trial. JAMA. 1999; 282(16):1554-60.

6. U.S. DEPARTMENT OF HEALTH AND HUMAN SERVICES. Dietary Guidelines for Americans, 2005. Disponivel em: http://www.healthierus.gov/dietaryguidelines. Acessado em: 15/08/2005.

7. Lind E, Joens-Matre RR, Ekkekakis P. What intensity of physical activity do previously sedentary middle-aged women select? Evidence of a coherent pattern from physiological, perceptual, and affective markers. Prev Med. 2005; 40(4):407-19.

8. Ekkekakis P, Lind E. Exercise does not feel the same when you are overweight: the impact of self-selected and imposed intensity on affect and exertion. Int J Obes. 2006; 30(4):652-60.

9. Pintar JA, Robertson RJ, Kriska AM, Nagle E, Goss FL. The influence of fitness and body weight on preferred exercise intensity. Med Sci Sports Exerc. 2006;38(5):981-8. presente estudo, registrando respostas semelhantes para PSE entre os grupos PN e SP (PSE 10), assim como Hills et al. ${ }^{(10)}$, que ao comparar mulheres PN e OB, não verificaram diferenças significantes na PSE. Esses resultados consistentemente confirmam os achados de Robertson et al. ${ }^{(18)}$, de que uma mínima diferença é observada para PSE em uma mesma intensidade relativa de trabalho $\left(\mathrm{FC}_{\text {máx }} \% \mathrm{VO}_{2 \text { máx }}\right)$, mas esta pode diferir quando o critério absoluto é adotado $\left(\mathrm{FC}_{1} \mathrm{VO}_{2}\right)$.

\section{CONCLUSÃO}

Os resultados do presente estudo demonstram que o grupo OB caminhou em velocidade inferior à reportada nos grupos PN e SP; contudo, respostas similares foram registradas para os parâmetros fisiológicos e, consequentemente, para as respostas perceptuais. Esses achados podem estar atrelados a ineficiência biomecânica de caminhada encontrada em sujeitos obesos durante a realização de atividades como essa. Os achados do presente estudo poderiam trazer contribuições teóricas e práticas. De um ponto de vista teórico, futuros delineamentos experimentais envolvendo autosseleção do ritmo de caminhada controlando a variável IMC devem ser considerados para confirmar os achados do presente estudo. De um ponto de vista prático, é verificado que a caminhada em ritmo autosselecionado proporciona um estímulo fisiológico adequado para a ocorrência de modificações orgânicas benéficas à saúde, independente do IMC, com respostas perceptuais similarmente baixas, as quais contribuiriam para menor probabilidade de abandono em programas de exercício físico. Os resultados da presente investigação devem ser analisados com cautela, visto que os parâmetros biomecânicos e o custo energético da caminhada não foram mensurados, e a extrapolação desses achados pode demonstrar resultados diferentes quando aplicados a outras populações (i.e., homens, sujeitos mais jovens ou com idade superior, níveis de condicionamento mais elevado, pacientes com condições especiais que limitam a prática de exercício e sujeitos com níveis de obesidade mais agravantes). Outro fator que deve ser considerado é a influência de mediadores psicológicos na autosseleção da intensidade de caminhada. Além disso, sugere-se que futuros estudos de intervenção envolvendo caminhada em ritmo autosselecionado devem ser realizados no intuito de melhor esclarecer a influência do IMC nas respostas fisiológicas e perceptuais.

\section{AGRADECIMENTOS}

Este trabalho foi parcialmente realizado com financiamento do Istituto per il Credito Sportivo (C. F. Buzzachera) e Coordenação de Aperfeiçoamento de Pessoal de Nível Superior (H. M. Elsangedy e K. Krinski).

Todos os autores declararam não haver qualquer potencial conflito de interesses referente a este artigo.

10. Hills AP, Byrne NM, Wearing $S$, Armstrong T. Validation of the intensity of walking for pleasure in obese adults. Prev Med. 2006; 42: 47-50.

1. Johnson JH, Phipps LK. Preferred method of selecting exercise intensity in adult women. J Strength Cond Res. 2006; 20(2): 446-449.

12. Borg GAV. Psychophysical bases of perceived exertion. Med Sci Sports Exerc. 1982; 14(5):377-81.

13. Noble BJ, Robertson RJ. Perceived Exertion. Champaign: Human Kinetics, 1996.

14. Mattsson E, Larsson UE, Rossner S. Is walking for exercise too exhausting for obese women? Int J Obes Relat Metab Disord. 1997; 21(5):380-6.

15. World Health Organization. Obesity: preventing and managing the global epidemic: Report of a WHO consultation. World Health Organ Tech Rep Ser. 2000; 894: i-xii, 1-253.

16. Durnin JV, Womersley J. Body fat assessed from total body density and its estimation from skinfold thickness: measurements on 481 men and women aged from 16 to 72 years. Br J Nutr. 1974; 32(1):77-97.

17. Siri WE. Body composition from fluid spaces and density. In: Brozek J, Henschel A. (Eds.). Techniques for measuring body composition. Washington: National Academy of Science, 1961;223- 44.

18. Robertson RJ, Moyna NM, Sward KL, Millich NB, Goss FL, Thompson PD. Gender comparison of RPE at absolute and relative physiological criteria. Med Sci Sports Exerc. 2000; 32(12):2120-29.

19. Cunningham DA, Rechnitzer PA, Pearce ME, Donner AP. Determinants of self-selected walking pace across age 19 to 66. J Gerontol. 1982; 37(4):560-564.

20. Browning CR, Baker EA, Herron JA, Kram R. Effects of obesity and sex on the energetic cost end preferred speed of walking. J Appl Physiol. 2006; 100(2):390-8. 